\title{
Preliminary update on structural and chemotaxonomical aspects of pharmacologically evaluated flavonoids
}

\author{
Sana Zouaoui (sanahamza971@gmail.com $)^{a}$, \\ Muhammad Farman (farman@qau.edu.pk) $)^{b}$ \\ Nabil Semmar (nabilsemmar5@gmail.com) $)^{a^{*}}$ \\ ${ }^{a}$ BioInformatics, bioMathematics \& bioStatistics Laboratory (BIMS). Pasteur Institute of Tunis. \\ University of Tunis El Manar. Tunisia. \\ ${ }^{b}$ Department of Chemistry, Quaid-i-Azam University, Islamabad-45320, Pakistan
}

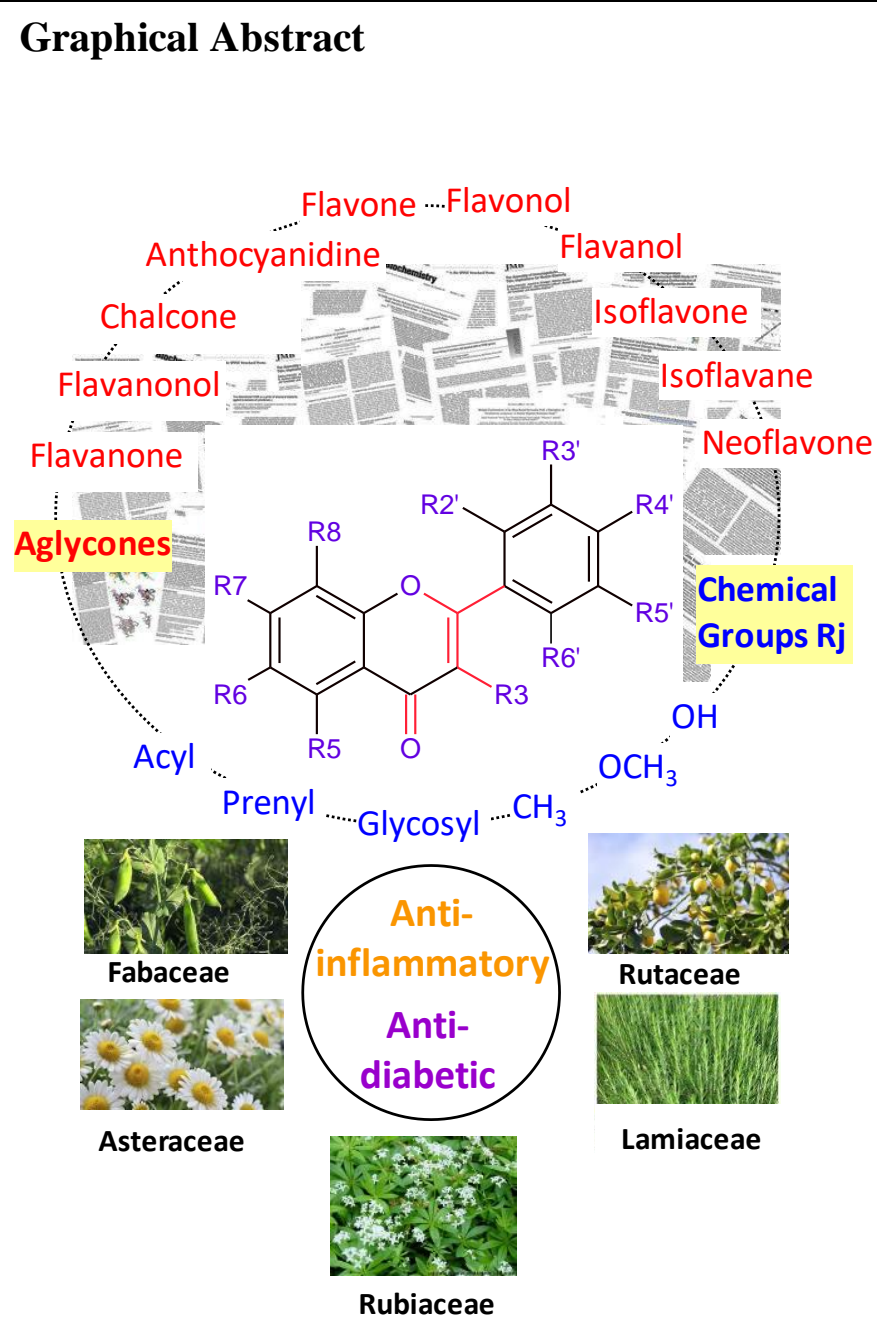

Abstract. This communication presents a preliminary update on pharmacologically evaluated flavonoids under several aspects including: (i) structure-structure associations, (ii) structure-plant affiliations and (iii) structureactivity trends. Although pharmacological evaluations are continuously performed on secondary metabolites, a small fraction of published (elucidated) molecules were revealed to be concerned with lab testing. This resulted in strongly sparse information in literature that did not favor integrative work in this field. While sparse data don't favor intensive analysis of well target system (e.g. a given pharmacological activity of a given metabolic family associated with a given plant taxon), big sets of cumulated data from many origins provide alternative way for extensive analysis helping to highlight backbone information on target biochemical system. This principle was applied to highlight different structural, functional and chemotaxonomical aspects of pharmacologically evaluated flavonoids. A number of 285 pharmacologically evaluated flavonoids (collected in bibliography) was considered. (i) Significant variations of relative frequencies of different chemical groups $\left(\mathrm{OH}, \mathrm{OCH}_{3}\right.$, glycosyls, 
prenyl) were highlighted in different flavonoid aglycones. (ii) Different plant families were revealed to be characterized by clear structural differentiation ways based on relatively high frequencies of some aglycones and chemical groups. (iii) Different chemical groups occurring in some carbons of aglycones showed positive or negative effects on anti-inflammatory and antidiabetic activities (benefitting from more available data).

Introduction. This communication presents a preliminary update on structural and functional order of pharmacologically evaluated flavonoids. It is a preliminary original work providing an initial statistical step for further valorization of sparse information available in bibliography. Such a sparse aspect represented a constraint toward integrative studies until now. However, extensive cumulation of pharmacological data from many published sources provides an alternative efficient way to openly extract evolving backbone information in this widely promising field.

Materials and Methods. Structure-Structure, structure-plant family and structure-activity trends were analyzed from 285 pharmacologically evaluated flavonoids belonging to different aglycone families and originated from different plant taxa (Mirossay et al., 2018; Cirmi et al., 2016; Panche et al., 2016; Xiao, 2015; Lago et al., 2014; Kumar et Pandey, 2013; Semmar, 2010). Nonparametric comparison and link tests as well as multivariate analyses were applied to highlight/detect such trends (Semmar, 2011, 2013).

Results. For structure-structure associations, $\mathrm{OH}$ and $\mathrm{OCH}_{3}$ showed opposite distributions of relative occurrences in overall chemical structural pool (Fig. 1a). Prenylation showed relatively higher occurrence in upstream flavonoids (chalcones, flavanones) and their immediate derivatives (isoflavanes, isoflavones). Glycosylation (Gly) showed lower occurrence than other chemical substitutions; this could be linked a priori to lower testing trend of glycosides because of well-known negative effect of Gly on pharmacological activities. For structure-plant family affiliations, relatively high occurrences were recorded for (i) flavanones and flavones in Rutaceae, (ii) isoflavones and isoflavanes in Fabaceae, (iii) flavones in Lamiaceae, (iv) flavones and flavonols in Asteraceae, (v) neoflavones in Rubiaceae (Fig. 1b). Moreover, Fabaceae showed more heterogeneous phenolic profile than the other plant families. Plant families were also differentiated by relative occurrences of chemical groups: $\mathrm{OH}$ was relatively more frequent in Asteraceae, Fabaceae and Lamiaceae whereas $\mathrm{OCH}_{3}$ markedly characterized Rutaceae and Rubiaceae. Concerning pharmacological activities, antidiabetic $(A D b)$ and anti-inflammatory (AInf) seemed to be influenced by types and positions of chemical groups, respectively (Fig. 1c): $A D b$ seemed to be favored by hydroxylation and glycosylation vs opposite effect from $\mathrm{OCH}_{3}$. However, AInf showed positive links with 6- $\mathrm{OCH}_{3}$ and 7-OH vs negative effects from 8-OH, and 8- $\mathrm{OCH}_{3}$.

Conclusion. This preliminary work showed highly informative aspect of pharmacologically evaluated flavonoids in matter of (i) chemical differentiation of plant families and (ii) links between structural traits and biological activities. The results call for further investigations in pharmacological evaluations of flavonoids. They are openly ready for updates by considering further biologically evaluated flavonoids as they are published. 
(a)

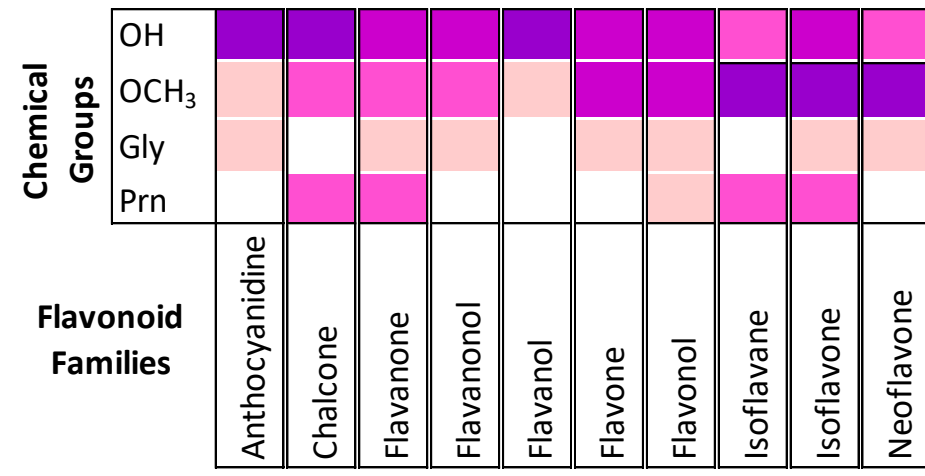<smiles>OC1Cc2ccccc2OC1c1ccccc1</smiles>

Flavanol

Dominant

Important

Frequent

Neoflavone
Isoflavone
Isoflavane
Flavonol
Flavone

(b)<smiles>O=C(C=Cc1ccccc1)c1ccccc1</smiles>

Chalcone

\section{Flavanol}

Flavanone

Chalcone

Catechine

Anthocyanidine

Botanical

Families
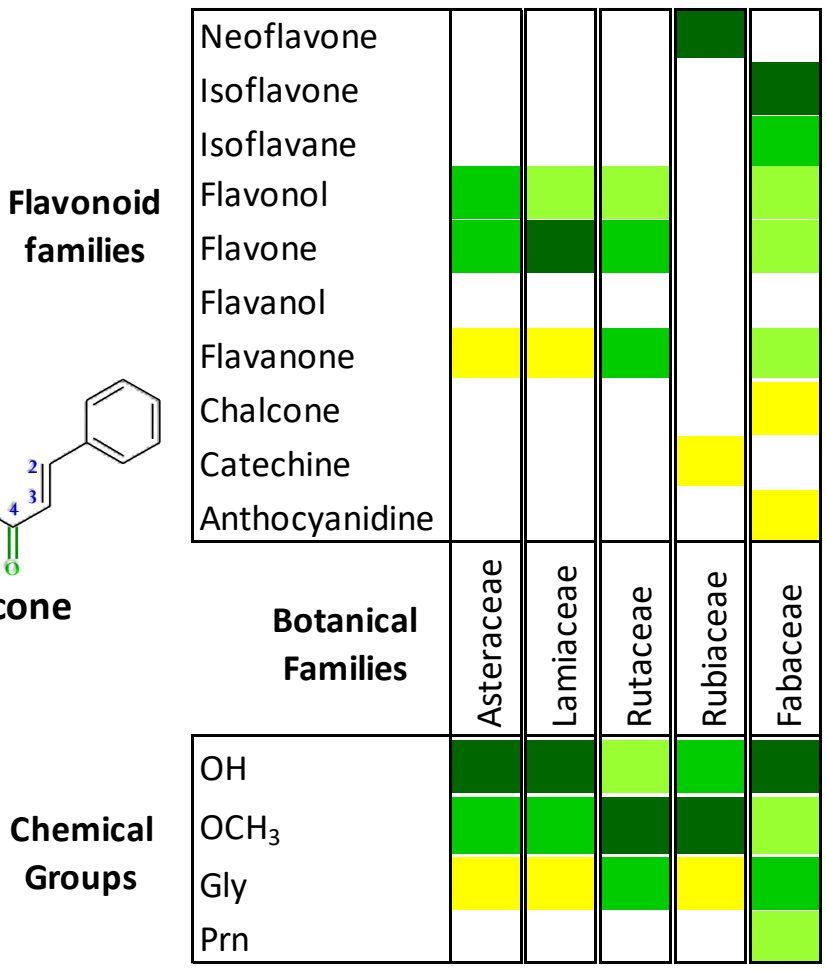

Dominant

Important

Frequent

Minor

Minor

Flavanone<smiles>COc1cccc(-c2cc(=O)oc3ccccc23)c1-c1ccccc1</smiles>

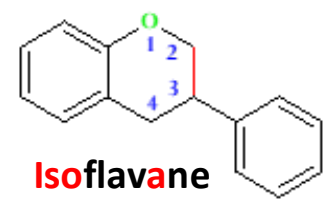

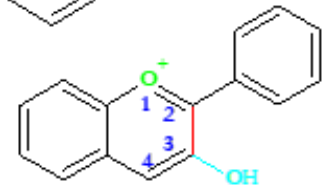

Anthocyanidin

(c) Anti-inflammatory activity

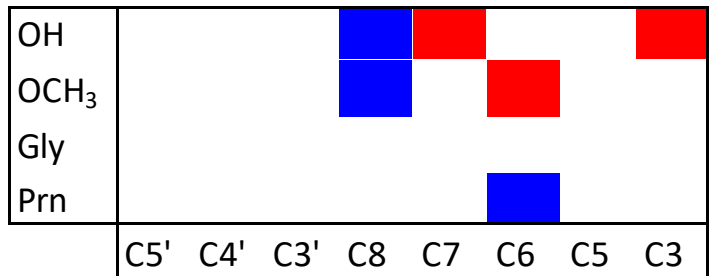

Substituted carbons of flavonoids

\section{Antidiabetic activity}

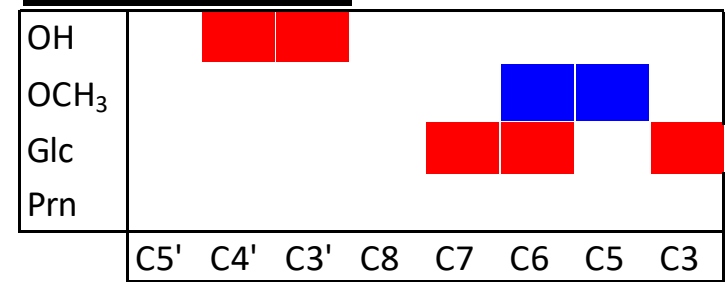

Substituted carbons of flavonoids

Positive Effect

Negative Effect

Figure 1. (a) Relative occurrence levels of chemical groups in different flavonoid families. (b) Relative occurrence levels of aglycones in different plant families. (c) Statistically detected chemical groups and substitution positions influencing anti-inflammatory and anti-diabetic activities of flavonoids. 


\section{References}

Cirmi S, Ferlazzo N, Lombardo GE, Ventura-Spagnolo E, Gangemi S, Calapai G, Navarra M, 2016. Neurodegenerative Diseases: Might Citrus Flavonoids Play a Protective Role?. Molecules, 21, 1312.

Kumar S, Pandey AK, 2013. Chemistry and Biological Activities of Flavonoids: An Overview, The ScientificWorld Journal, 2013, 1-16.

Lago JHG, Toledo-Arruda AC, Mernak M, Barrosa KH, Martins MA, Tibério IFLC, Prado CM, 2014. Structure-Activity Association of Flavonoids in Lung Diseases , Molecules, 19, 3570-3595.

Mirossay L, Varinská L, Mojžiš J, 2018. Antiangiogenic Effect of Flavonoids and Chalcones: An Update, International Journal of Molecular Sciences, 19, 27.

Panche AN, Diwan AD, Chandra SR, 2016. Flavonoids: an overview , Journal of Nutritional Science, 5, e47, 1-15.

Semmar N, 2010. Chemotaxonomical analyses of herbaceous plants based on phenolic and terpenic patterns. Nova Science Publishers, New York, 257p.

Semmar N, 2011. Computational metabolomics. Nova Science Publishers, New York, 238p.

Semmar N, 2013. Native statistics for natural sciences. Nova Science Publishers, New York, 515p.

Xiao J, 2015. Dietary Flavonoid Aglycones and Their Glycosides: Which Show Better Biological Significance? , Critical Reviews in Food Science and Nutrition, 57(9), 1874-1905.

Xiao J, Capanoglu E, Jassbi AR, Miron A, 2015. Advance on the Flavonoid C-glycosides and Health Benefits , Critical Reviews in Food Science and Nutrition, 56:sup1, S29-S45 